\title{
Über Methoden und Fehlerquellen der Versuche über künstliche Parthenogenese.
}

\author{
Von \\ Jacques Loeb. \\ (From the Hull Physiological Laboratory of the University of Chicago.)
}

Eingegangen am 24. Oktober 1901.

1) Auf Grund ron verschiedenen Mitheilungen europäischer Autoren, welche Schwierigkeiten fanden bei der Wiederholung oder Weiterführung meiner Versuche iiber künstliche Parthenogenese, möchte ich einige Bemerkungen über die Methode und Fehlerquellen dieser Versuche hier mittheilen. Auf die Nothwendigkeit der Sterilisation des Seewassers, der Instrumente, Hände und der Thiere selbst brauche ich wohl nicht einzugehen, sie ist selbstverständlich. Nur die größte Fehlerquelle möchte ich in diesem Zusammenhang erwähnen, nämlich die Neigung der Männchen, namentlich bei reifen Seeigeln, das Seewasser in Eimer, in dem sie ins Laboratorium gebracht werden, mit Sperm zu füllen. Es ist rathsam, die Weibchen erst 24 Stunden oder wenn möglich länger in spermfreiem Seewasser isolirt zu halten, ehe sie zu Versuchen ihrer künstlichen Parthenogenese benutzt werden. Hat man die nöthigen Vorsichtsmaßregeln g'egen Infektion durch Sperm getroffen, so ist der nächste Schritt, die unbefruchteten Eier zur Entwickelung zu bringen. Bei den Eiem von Seeigeln ist die einzige wirksame Methode, die bis jetzt bekannt ist, dieselben für ungefähr $1 \frac{1}{2}$ Stunden in Seewasser zu bringen, dessen osmotischer Druck um einen bestimmten Betrag erhöht ist. Im Princip ist es gleichguiltig, wie diese Druckerhöhung erfolgt, durch Verdampfen von Wasser oder durch Zusatz von Salz oder Zucker oder Harnstoff zum Wasser. Für denjenigen aber, der recht viele und möglichst normale Larven erhalten will, ist die Wahl der 
Methoden etwas beschränkter. Ich finde nach all meinen Erfahrungen den Zusatz von Kaliumsalzen und Natriumsalzen, namentlich Kaliumchlorid und Natriumchlorid, vielleicht am günstigsten. Die Quantität der Koncentrationserhöhung ist von entscheidender Bedeutung. Wenn man die richtige Koncentration nicht genau trifft, so wird man Misserfolge haben, und es ist erstaunlich, wie sehr die nöthige Koncentration in verschiedenen Versuchsreihen schwankt. Ob die Schwankungen aussehließlich individueller Natur sind und dem verschiedenen Reifegrad der Eier entsprechen, vermag ich nicht anzugeben. Vielleicht hat auch die Temperatur einen Einfluss. Um all solchen $\mathrm{Zu}-$ fälligkeiten zu entgehen, arbeite ich stets mit einer Reihe von Lösungen. Auf diese Weise bin ich sicher, wenigstens in einer der Lösungen gute Resultate zu erzielen. Als »stock solution « benutze ich eine $2 \frac{1}{2}$ n. KCl-Lösung, d. h. eine Lösung, die ungefähr $186 \mathrm{~g}$ KCl im Liter Wasser hat. In meinen Versuchen waren die Lösungen genau titrirt, aber das ist für die meisten Zwecke überflüssig. Ich nehme sechs Gefäße, jedes mit $100 \mathrm{ccm}$ Seewasser, und füge diesen sechs Gefäßen der Reihe nach 8, 10, 12, 14, 16, $18 \mathrm{ccm}$ der obigen $21 / 2$ n. KCl-Lösung zu. Die unbefruchteten Seeigeleier werden nuu in diese sechs Gefäße (und außerdem in ein Kontrollgefäß mit reinem Seewasser) vertheilt. Auch in Bezug auf die Zeit, während der die Eier in diesen Lösungen verweilen müssen, bestehen Verschiedenheiten. Es ist desshalb geboten, die Eier nicht alle auf einmal, sondern in verschiedenen Intervallen herauszunehmen, etwa nach $1 / 2$, $1,1 \frac{1}{2}$ und 2 Stunden. Auf diese Weise wird man die optimale Koncentration und Versuchsdauer sicher treffen. Chlorkalium hat den Vortheil, dass es meist zur Bildung eines einzigen Embryos aus je einem Ei führt, während bei der Anwendung von Natriumchlorid Polyembryonie die Regel ist. Aber möglicher Weise fällt die Skeletbildung bei der Anwendung der Natriumsalze besser aus als bei Anwendung der Kaliumsalze. Herr Hunres erlangte in Woods Holl sehr gute parthenogenetische Plutei mit Seewasser, dessen Koncentration durch Verdampfen um ca. $30-40 \%$ erhöht worden war. Wurden die Eier ca. 1-2 Stunden in solches Seewasser gebracht, so entwickelten sie sich sehr schön, wenn sie in normales Seewasser zurïckgebracht wurden.

Ein zweiter wichtiger Umstand, der bei diesen Versuchen vielleicht eine Rolle spielt, ist die Temperatur. Die Versuche in Woods Holl (sowie Wilsox's Versuche in Beaufort) sind alle bei Sommertemperatur angestellt und die Temperatur des Wassers war $20^{\circ}$ Celsius 
oder darüber. In Californien sehwankte die Temperatur in meinen Versuchen erheblich, sie war oft ziemlich niedrig und es gelang mir gelegentlich nicht, künstliche Parthenogenese herbeizuführen. Ich bezog das damals auf unvollkommene Reife der Eier. Vielleicht war das richtig und vielleicht erklärt das manche der negativen Resultate europäischer Forscher, welche im Winter arbeiteten. Allein ich habe seitdem daran gedacht, ob nicht am Ende die Temperatur in der Weise die Versuchsresultate beeinflusst, dass unterhalb einer bestimmten Temperatur die kïnstliche Parthenogenese nicht oder nur, sehwer zu Stande kommt. Ich werde darin bestärkt durch eine briefliche Yittheilung, die mir Herr DoNCaster machte, der in Neapel gearbeitet hat, nämlich dass er erst nur negative Resultate erbielt, dass er dann aber anf die Vermuthung kam, dass die Temperatur des Wassers in Neapel zu niedrig sei und dass er die Versuche in Wasser von ungefähr $20^{\circ}$ anstellte. Im letzteren Falle fielen die Versuche positiv aus.

2) Das bisher Gesagte bezieht sich nur auf Versuche mit Seeigeleiern, besonders Arbacia. Ganz besondere Vorsichtsmaßregeln sind erforderlich, wenn man mit Seesterneiern arbeitet. A. Mathews hat die Beobachtung gemacht, dass man die in Seewasser zur Reife gekommenen unbefruchteten Eier von Seesternen (Asterias) dadurch zur Entwickelung bringen kann, dass man sie schüttelt und dass ein Zeitpunkt existirt, bei dem die mit dem Überbringen der Eier ans einem Gefäß in ein anderes verbundene Erschütterung ausreicht, Larven zu erzielen. Eier mit diesem Grad der Empfindlichkeit erfordern zwei Vorsichtsmaßregeln, wenn man nicht den gröbsten Täuschungen anheimfallen will. Erstens ist es nöthig, die Eier in solcher Weise aus einem Gefäß in ein anderes zu bringen, dass dabei jede mechanische Erschiitterung ausgeschlossen ist. Am besten erzielt man das durch Benutzung von Pipetten mit sehr weiter öffnung zum Aufsaugen und Übertragen der Eier. Die letzteren Manipulationen müssen dann mit der größten Vorsicht geschehen. Die zweite Vorsichtsmaßregel besteht darin, dass man jedes Mal, wenn man die Versuchseier aus einer Lösung in eine andere oder in Seewasser zuriuckbringt, auch dieselbe mechanische Manipulation in genau derselben Weise mit den Kontrolleiern vornimmt. In dieser Weise lässt es sich entscheiden, ob die parthenogenetische Entwickelung im einzelnen Falle anf mechanisehe Erschütterung zurückznführen ist oder anf andere Agentien, die man anwendet. Mit diesen Cautelen haben wir eine Reihe von Versuchen diesen Sommer an Asterias-Eiern 
durchgeführt und bis jetzt gefunden, dass, abgesehen von der mechanischen Ersehiitterung, nur zwei Methoden zur kiunstlichen Parthenogenese der Seesterneier führen, nämlich erstens Einbringen der Eier für 3-20 Minuten in Seewasser, dem man 3-5 cem einer $\frac{\mathrm{n}}{10} \mathrm{HCl}-$ oder irgend einer anderen inorganischen Säurelösung zu je $100 \mathrm{ccm}$ zufügt. Die zweite Methode, die mein Schüler Mr. A. W. Greelex fand, besteht darin, dass die Eier, nachdem sie eine bestimmte Zeit in Seewasser gelegen haben, für eine Reihe von Stunden auf Eis gebracht werden. Andere Methoden ergaben dagegen alle negative Resultate, namentlich das Erwärmen der Eier, das Mr. Greelex ebenfalls versuchte. Es gelang uns auch nicht durch Wasserentziehung klare Resultate zu erzielen, so dass ich vermuthe, dass vielleicht in meinen früheren Versuchen, bei denen ich durch Koncentrationserhöhung von Seewasser Seesterneier zur Entwickelung kommen sah, mechanische Erschïtterungen die Entwickelung hervorriefen. Ich glaube auch, dass DeLaGe dureh diesen Umstand theilweise irregeführt worden ist, wenn er behauptet, dass es sich bei den Mitteln zur künstlichen Parthenogenese nur um eine »excitation banale * handele, die sehr verschiedenartig sein könne und er giebt an, dass ungefähr jeder physikalische oder chemische Eingriff künstliche Parthenogenese herbeiführt. Ich glaube nicht, dass er eine solche Behauptung auf Grund von Versuchen an Seeigeleiern aufgestellt haben wirde, und ich vermuthe, dass er auch bei Seesterneiern nur desshalb zu diesem Ausspruch gefuihrt worden ist, weil er in Wirklichkeit, ohne sich dessen bewusst zu sein, die Eier in einem Theil seiner Versuche durch denselben Eingriff, nämlich mechanische Erschittterung, zur Entwickelung brachte. Bei Seeigeleiern wirkt die Erschütterung nicht in dieser Weise und diese Fehlerquelle, welche beim Arbeiten mit Seesterneiern so unangenehm ist, existirt hier nicht. Gleichwohl hatte ich es von vorn herein mir zur Regel gemacht, die Kontrolleier auch bei Versuchen mit Seeigeleiern mechanisch stets gleich zu behandeln wie die eigentlichen Versuchseier.

3) Die für Versuche an Seesterneiern nöthigen Vorsichtsmaßregeln miissen auch bei Versuchen mit den Eiern von Anneliden, Chaetopterus und Amphitrite, benutzt werden. In beiden Formen ist es uns diesen Sommer gelungen, kiinstliche Parthenogenese dureh Schütteln und mechanische Erschütterung der Eier herbeizuführen, bei Chaetopterus ist dieses Resultat aber beschränkter und unsicherer als bei Amphitrite. Lässt man die unbefruchteten Amphitriteneier 
in natürlichem Seewasser liegen ohne das Gefäß zu erschüttern, so entwickeln sich die Eier ebenso wenig zu Larven wie bei Seesternen. Von einer »natürlichen « Parthenogenese dieser Formen zu sehwimmenden Larven ist keine Rede. Lässt man sie aber 30 Minuten im Seewasser liegen, so können die unbefruchteten Amphitriteneier dadurch zur Entwickelung zu Larven gebracht werden, dass man sie aus einem Gefäß mittels einer Pipette in ein anderes Gefäß spritzt. Das gelingt zwar nicht bei jeder Kultur, aber doch sehr bäufig. Dagegen gelingt es, die unbefruchteten Eier von Amphitrite jedes Mal und ohne jede Erschiitterung zur Entwickelung zu bringen, wenn man sie in Seewasser bringt, dem man eine kleine, aber bestimmte Menge eines löslichen Calciumsalzes hinzufügt. Es ist nicht nöthig, die Amphitriteneier aus einer solchen Lösung in Seewasser zurïckzubringen, sie entwickeln sich in einer solchen Lösung zu schwimmenden Larven. Wie also Wasserstoffionen die Larvenentwickelung yon Seesterneiern herbeiführen, so führen Calciumionen die Entwickelung ron Amphitriteneiem herbei. Der Zusatz von 2 bis 5 ecm einer normalen Calciumnitrat- oder Calciumehloridlösung zu $100 \mathrm{ccm}$ Seewasser genügt für diesen Zweck.

Meine früheren Versuche über die specifischen Wirkungen von Kaliumionen auf die Entwickelung unbefruchteter Chaetopterus-Eier habe ich dieses Jahr wiederholt und bestätigt gefunden. Diese Eier entwickeln sich, wenn man dem Seewasser eine kleine, aber bestimmte Yenge irgend eines löslichen Kaliumsalzes $\left(\mathrm{KCl}, \mathrm{KNO}_{3}, \mathrm{~K}_{2} \mathrm{SO}_{4}\right) \mathrm{zu}-$ fuigt (ca. 1-2 cem einer 21/2 n.-Lösung eines dieser Salze zu $100 \mathrm{cem}$ Seewasser). Es ist nicht nöthig, die Eier aus dieser Lösung herauszunehmen und in normales Seewasser zurückzubringen. Es mag vielleicht. besonders betont werden, dass Calcium- und Kaliumionen keine specifische Wirkung auf die Seesterneier haben, dass Kaliumionen ebenso nicht im Stande sind, die Amphitriteneier zur Entwickelung zul bringen und dass Calciumionen bei Chaetopterus-Eiern wirkungslos bleiben.

4) Indirekt sind noch folgende Thatsachen ron Bedeutnng für die Methode der Versuche über künstliche Parthenogenese. Alle die Ionen, welche bei Seesternen, Amphitrite und Chaetopterus Parthenogenese herbeiführen, veranlassen anch gleichzeitig Agglutination der betreffenden Eier und Bildung von Riesenembryonen. Das Problem, das DRIESCH sich einst vorsetzte und das bei Seeigeln auf große Schwierigkeiten stößt, nämlich den Inhalt mehrerer Eier zum Verschmelzen zu bringen, gelingt in diesen Versuchen spielend und im 
großartigsten Maßstabe, ganz besonders bei den Eiern von Seesternen. Ich brauche kaum zu betonen, dass es von großer theoretiseher Bedeutung ist, dass die Ionen, welche künstliche Parthenogenese herbeiführen, auch gleichzeitig den physikalischen Zustand der Eier in bestimmtem Sinne ändern. Es ist mir noch nicht gelungen, ein specifisches Ion zu finden, dass die unbefruchteten Eier von Seeigeln zur Entwickelung bringt. Es wäre von Interesse festzustellen, ob ein solches Ion, wenn es gefunden wird, ebenfalls eine Agglutination der Seeigeleier veranlasst.

An anderen Formen, Nereis, Podarke, Phascolosoma, sind die Versuche so weit geführt, dass wir behaupten können, künstliche Parthenogenese (schwimmende Larven) ist bei denselben möglich. Die Versuche sind aber nicht allseitig genug durchgearbeitet, um veröffentlicht werden zu können. Von den hier mitgetheilten Methoden können wir mit Bestimmtheit behaupten, dass sie an den amerikanischen Formen des atlantischen Oceans zum Ziele fuihren. Für die Auffindung weiterer Methoden diurfte es sich vielleicht empfehlen, folgende (theoretische) Gesichtspunkte im Ange zu behalten, die ich in verschiedenen friiheren Arbeiten ansfiihrlicher besprochen habe. Die betreffenden künstlichen Eingriffe zur Erzielung ron Parthenogenese müssen darauf hinauslaufen, dass sie erstens die Verfliussigung oder sonstige Zerstörung der Kernmembran begiunstigen; dass sie zweitens auch die physikalischen Eigenschaften des Protoplasmas (Viscosität etc.) in bestimmtem Sinne ändern. Es scheint, dass in den Eiern, an denen bis jetzt die künstliche Parthenogenese gelungen ist (und vermuthlich an vielen, wenn nicht allen sonstigen Eiern), unter natiurlichen Umstïnden im unbefruchteten Ei solche chemische Vorgänge ablaufen, die das Ei im Sinne der zwei erwähnten Bedingungen zu verändern streben, dass diese aber unter gewöhnlichen Umständen so langsam ablaufen, dass das $\mathrm{Ei}$ abstirbt, ehe es zur wirklichen Zelltheilung kommt. Solche Umstände, welche den Ablauf dieser natürlichen Processe zu beschleunigen im Stande sind, werden auch das unbefruchtete Ei zur Entwickehung veranlassen. 